\title{
Study on Fundamental Technology of Multi Directional Vibration Piezoelectric Power Generation
}

\author{
Arslan Habib $^{1 *}$, Adesh Ananta ${ }^{2}$ \\ ${ }^{1}$ Northwestern Polytechnical University, Xi'an, China \\ ${ }^{2}$ State Grid Tianjin Energy Saving Service Co., Ltd., Tianjin, China \\ Email: Arslan.2010@live.com,2253694671@qq.com
}

How to cite this paper: Habib, A. and Ananta, A. (2018) Study on Fundamental Technology of Multi Directional Vibration Piezoelectric Power Generation. World Journal of Engineering and Technology, 6, $1-16$.

https://doi.org/10.4236/wjet.2018.61001

Received: May 11, 2017

Accepted: December 23, 2017

Published: December 26, 2017

Copyright ( $) 2018$ by authors and Scientific Research Publishing Inc. This work is licensed under the Creative Commons Attribution International License (CC BY 4.0).

http://creativecommons.org/licenses/by/4.0/

(c) (i) Open Access

\begin{abstract}
The problem of low vibration energy collection efficiency in the environment is the focus of the current piezoelectric power generation technology. In order to improve the collection efficiency of vibration energy in the environment, a key technology of piezoelectric power generation is studied, which can collect the vibration energy of multiple directions in the environment. Firstly, the mathematical model of piezoelectric cantilever beam is established by using the basic theory of piezoelectric power generation technology, and the vibration mechanics analysis of the piezoelectric cantilever beam is carried out. Then, the finite element simulation of the piezoelectric cantilever beam is carried out by using ANSYS, and the natural frequency is consistent with the ambient vibration/environment frequency; finally, multi-directional piezoelectric power generation device is made, and the theoretical analysis and experimental test are carried out. The experimental results show that the key technology of multidirectional vibration piezoelectric power generation can effectively improve the collection efficiency of vibration energy in the environment.
\end{abstract}

\section{Keywords}

Multi-Directional, Vibration Energy, Structure Optimization, Collection Efficiency

\section{Introduction}

Micro-electromechanical systems have been widely used in field animal GPS tracking monitoring, embedded systems, health monitoring, national defense 
security application systems and environmental monitoring, etc. [1], but the power supply has been hindering its development. At present, researchers all over the world focus on collecting energy from the environment for the power supply of micro electro mechanical systems [2] [3]. MAS (Multi-Agent System) is a kind of artificial intelligence computing method; its independent characteristics make it possible for electronic devices with common or conflicting goals to coexist in complex power systems. MAS based on JADE (Java Agent Development Framework) platform for Agent information interactive, distributed simulation based on JADE simulation platform has become an important research direction in recent years [4].

With the continuous development of energy harvesting technology, piezoelectric power generation technology has become the focus of research in the field of Micro-electromechanical (MEMS) power supply [5]-[11]. In 2003, at University of Virginia, Henry A. Sodano studied the bimorph structure of piezoelectric cantilever beam and Donald J. studied the piezoelectric cantilever beam [12] [13], When the device is applied with $50 \mathrm{~Hz}$ sinusoidal vibration, a $3 \mathrm{~V}$ voltage can be output. South Korean scientists Ji-Yoon Kang and Hyung-Jun Kim [14] designed a piezoelectric cantilever vibration energy-harvesting device, which produces voltage by deforming it with a certain intensity load. In 2006, Jilin University had designed a press disc piezoelectric power generation device; this device has been successfully used in microelectronic devices. In order to improve the energy collection efficiency of piezoelectric devices, Yuan Jiangbo of Harbin Institute of Technology has developed a composite piezoelectric cantilever beam. The output energy can be used to supply power for MEMS devices [15]. Energy storage devices are arranged in the non-grid-connected wind power generation system to ensure the power quality, and it has become the key to full utilization of renewable energy. In the case of wind speed fluctuation, the DC bus control strategy of the wind turbine is proposed in this paper. It can reduce the impact on the unit converter and the power load; this ensures safe and stable operation of non-grid connected wind turbines [16].

Through investigation, the piezoelectric power generation technology is based on direct piezoelectric effect, excluding the reverse piezoelectric effect [17] [18], but the direction selectivity of the unidirectional piezoelectric device is stronger and the change of vibration direction in the environment greatly reduces the collection efficiency of the power generation device. In view of the above problems, the influence of the direction selectivity of piezoelectric devices and the variability of environmental vibration direction is fully considered in this paper, the key technology of multidirectional vibration piezoelectric power generation is studied in this paper to improve the efficiency of vibration energy collection in power plant.

\section{Key Technical Principle of Multi-Directional Vibration Piezoelectric Power Generation}

In this paper, the multi-directional vibration piezoelectric power generation 
technology is a multi-directional vibration piezoelectric power generation device, the structure design is as follows: A multidirectional piezoelectric generator is composed of five identical piezoelectric cantilever structures with a mass block, a support body and a fixed base. Five identical piezoelectric cantilever beams are equally fixed on the support like a fan; the geometry model is shown in Figure 1. The principle is: when the device is excited by a certain direction. Due to force, when the piezoelectric cantilever will vibrate in this direction, the vibration energy collection can be achieved. When the excitation direction changes, the piezoelectric cantilever beam on the device always has vibration on the cantilever beam in the corresponding direction, Therefore, the vibration energy in the environment can be collected in a multi direction manner, and the collection efficiency of the piezoelectric device can be improved.

The direction of vibration in the environment is changeable, and the direction selectivity of the unidirectional power generation device is stronger. In this paper, the key technology of multidirectional vibration piezoelectric power generation breaks the traditional one way of collecting vibration, the vibration energy in the environment is collected from multiple directions. When the vibration direction changes, the cantilever beam in the corresponding direction generates vibration to collect the vibration energy in the environment. Therefore, as long as the optimization device is able to produce resonance, the multi-directional vibration piezoelectric power generation key technology can achieve the highest efficiency.

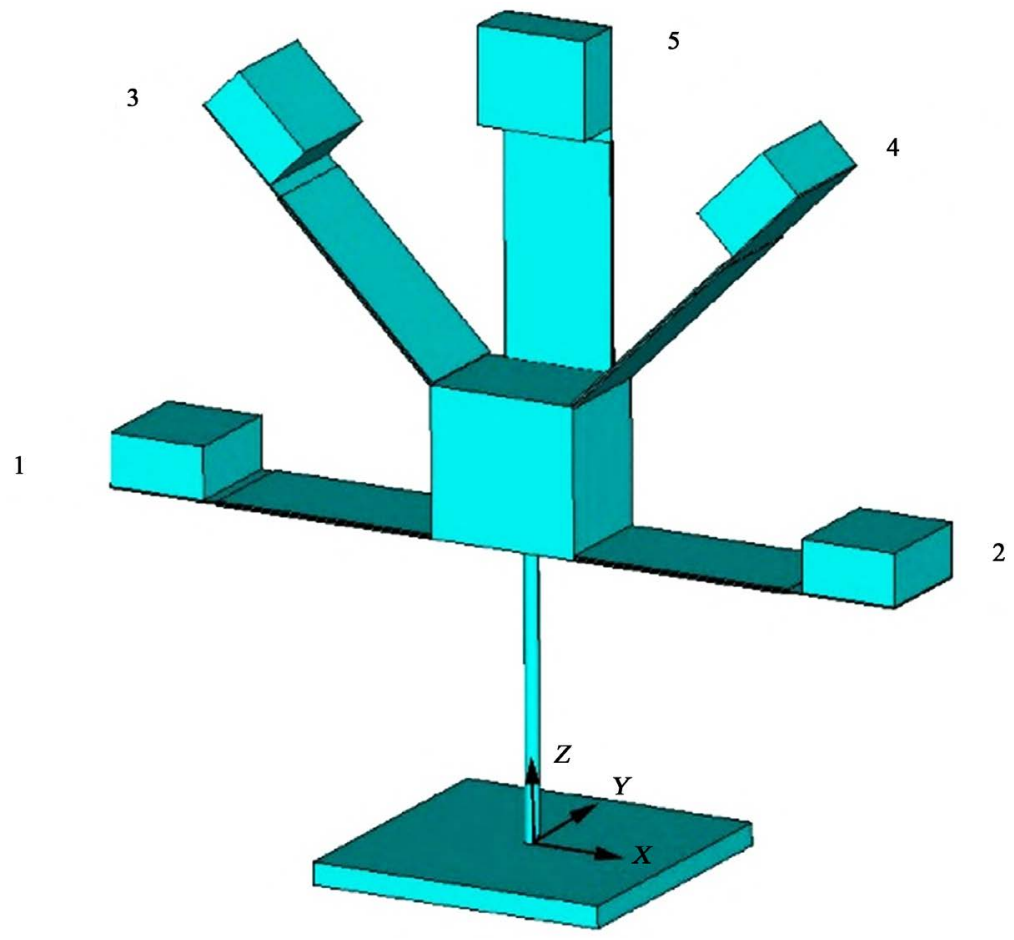

Figure 1. Multi-directional vibration piezoelectric power generation device. 


\section{Mathematical Modeling and Analysis of Piezoelectric Cantilever Beam}

The mathematical model of piezoelectric cantilever beam is established based on the power generation theory of piezoelectric power generation technology. In the mathematical modeling of the piezoelectric cantilever, one piezoelectric cantilever is selected as the research object. Figure 2 shows a pressure of a multidirectional piezoelectric generator Schematic diagram of an electric cantilever beam. We glued the piezoelectric ceramic on the substrate.

When the piezoelectric cantilever generator structure is forced to vibrate, the

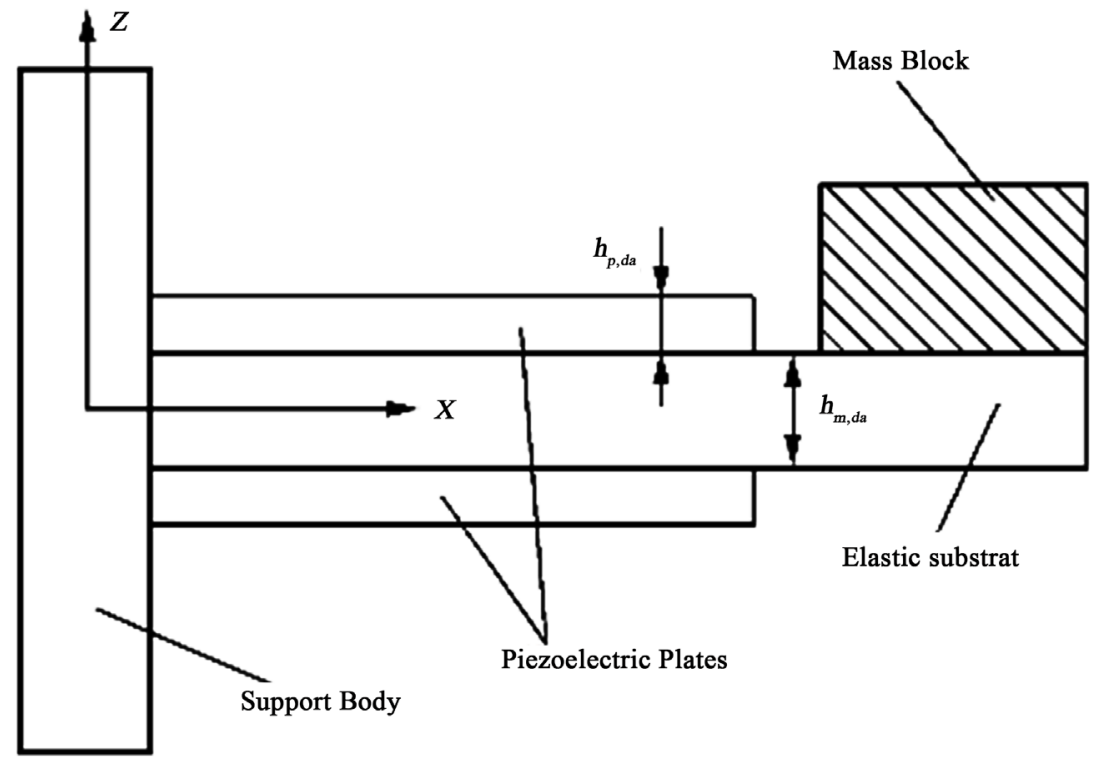

(a)

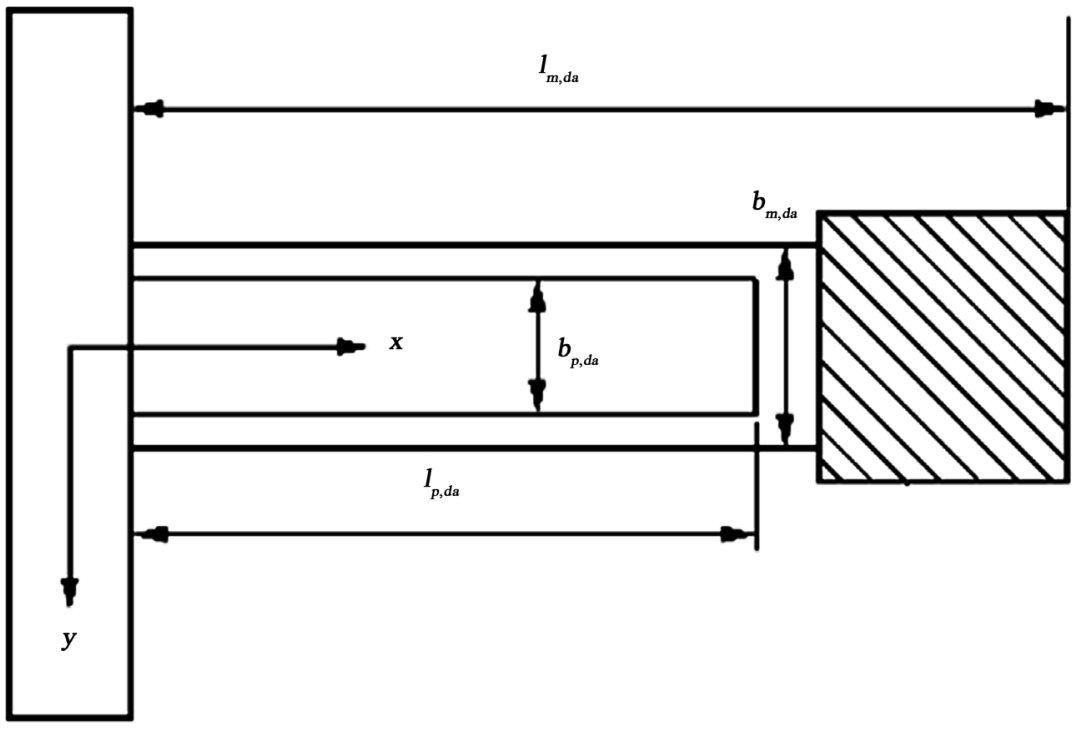

(b)

Figure 2. Schematic diagram of piezoelectric cantilever beam. (a) Main view; (b) Top view. 
vibration mechanics and open circuit voltage analysis are carried out. The piezoelectric cantilever power structure can be used with spring damping system as shown in Figure 3 and this is equivalent to the vibration model.

In the above vibration model, $k_{c a}$ represents the equivalent stiffness of the piezoelectric cantilever, the equivalent damping is expressed by $C_{c a}$, and the $m_{c a}$ is the lumped mass of the system. The relative displacement between mass and support is expressed by $y_{c a, o u}$, Displacement of vibration source is expressed by $y_{c a, i n}$. The theoretical model of piezoelectric cantilever beam is as follows [19]-[24].

$$
m_{c a} \ddot{y}_{c a, o u}+C_{c a} \dot{y}_{c a, o u}+k_{c a} y_{c a, o u}=-m_{c a} \ddot{y}_{c a, \text { in }}
$$

The vibration is analyzed; the absolute displacement of mass is $y_{c a, o u}+y_{c a, \text { in }}$, under the excitation of vibration source, the inertial force generated by mass block can be expressed as

$$
F_{c a}=m_{c a}\left(\ddot{y}_{c a, o u}+\ddot{y}_{c a, i n}\right)
$$

In the process of vibration of piezoelectric devices, the device converts mechanical energy into electrical energy. In this process, the open circuit voltage generated by the upper piezoelectric plate of the piezoelectric cantilever power generation structure is

$$
\begin{gathered}
V_{c a, 1}\left\{\begin{array}{c}
-\frac{1}{4 x_{1}} e_{31} b_{p, d a} l_{p, d a} F_{c a}\left(h_{p, d a}+h_{m, d a}\right)\left(2 l_{a d, d a}-l_{p, d a}\right) \\
{\left[\frac{1}{3 x_{1}} E_{p, d a} b_{p, d a} h_{p, d a}\left(h_{p, d a}^{2}+\frac{3}{4} h_{m, d a}^{2}+\frac{3}{2} h_{m, d a}\right)+1\right]}
\end{array}\right\} \\
{\left[\frac{1}{12 x_{1}^{2}} e_{31}^{2} E_{p, d a} b_{p, d a}^{3} l_{p, d a} h_{p, d a}\left(h_{p, d a}^{2}+\frac{3}{4} h_{m, d a}^{2}+\frac{3}{2} h_{m, d a} h_{p, d a}\right)\left(h_{p, d a}+h_{m, d a}\right)^{2}\right.} \\
+\frac{1}{2 x_{1}} e_{31}^{2} b_{p, d a}^{2} l_{p, d a}\left(h_{p, d a}+h_{m, d a}\right)^{2}+\varepsilon_{33} l_{p, d a} b_{p, d a} \frac{1}{h_{p, d a}}
\end{gathered}
$$

where: $\quad x_{1}=E_{p, d a} b_{p, d a}\left(\frac{2}{3} h_{m, d a}^{3} h_{p, d a}+h_{m, d a} h_{p, d a}^{2}\right)+\frac{1}{12} E_{m, d a} b_{m, d a} h_{m, d a}^{3} ; \quad b_{p, d a}, \quad h_{p, d a}$ The width and thickness of the elastic substrate, respectively; $l_{m, d a}, l_{m e, d a}$ The

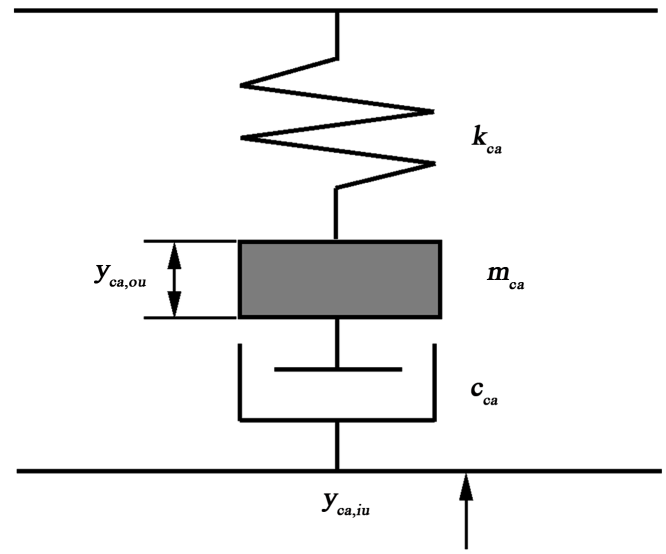

Figure 3. Equivalent model of cantilever beam vibration system. 
The width and thickness of the piezoelectric plate, respectively; $b_{m, d a}, h_{m, d a}$ length of the elastic substrate, the distance from the free end of the piezoelectric cantilever beam to the mass center of the mass block; $l_{a d, d a}$ and $l_{m, d a}$ is the sum of $l_{m e, d a} ; \varepsilon_{33}$ Dielectric constant of piezoelectric material; $e_{3,1}$ The intensity of electric field in piezoelectric layer on cantilever beam; $E_{m, d a}, E_{p, d a}$ The elastic modulus of the elastic substrate and the piezoelectric ceramic plate, respectively.

Similarly, the open circuit voltage produced by the lower lamination of the cantilever beam in the electromechanical conversion process $V_{c a, 3}=-V_{c a, 1}$.

\section{Finite Element Simulation Analysis of Piezoelectric Cantilever Beam}

Finite element simulation software ANSYS multi-physics coupling analysis module can do a good simulation of piezoelectric coupling field, to achieve mechanical energy and electrical coupling analysis. In the analysis of piezoelectric ceramic plates by ANSYS, the influence of the size of the elastic substrate and the parameters of the mass block on the output voltage and frequency of the piezoelectric cantilever beam is optimized.

\subsection{Finite Element Simulation Model of Piezoelectric Cantilever Beam}

PZT (Lead zirconate Titanate) is used to make ultrasound transducers and other sensors and actuators, as well as high-value ceramic capacitors and FRAM chips. The PZT piezoelectric ceramics were used as the initial piezoelectric cantilever beam, its length, width and thickness were $30 \mathrm{~mm}, 15 \mathrm{~mm}$ and $0.2 \mathrm{~mm}$, respectively. Beryllium bronze is used as material for metal elastic substrate. Its length, width and thickness are $45 \mathrm{~mm}, 15 \mathrm{~mm}$ and $0.2 \mathrm{~mm}$, respectively. Using iron blocks (mass) of length, width and thickness of $12 \mathrm{~mm}, 15 \mathrm{~mm}$ and $5 \mathrm{~mm}$, respectively. The finite element model of the piezoelectric cantilever beam is shown in Figure 4.

\subsection{Effect of Mass Thickness on Frequency}

In order to obtain the relationship between the mass size parameter and the natural frequency of the piezoelectric cantilever, the thickness of the mass is set to 4 - $8 \mathrm{~mm}$, and the other parameters are unchanged. Results as shown in Figure 5,

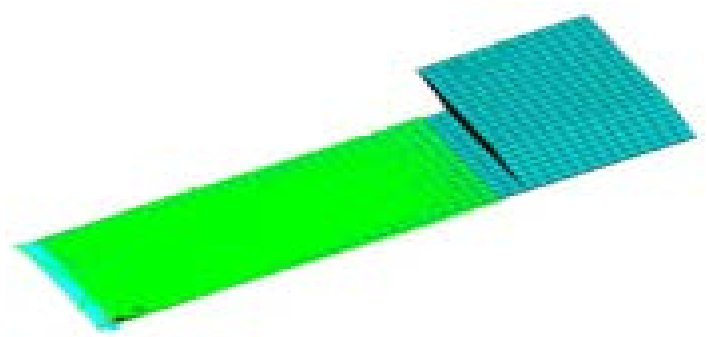

Figure 4. Finite element simulation model of piezoelectric cantilever beam. 


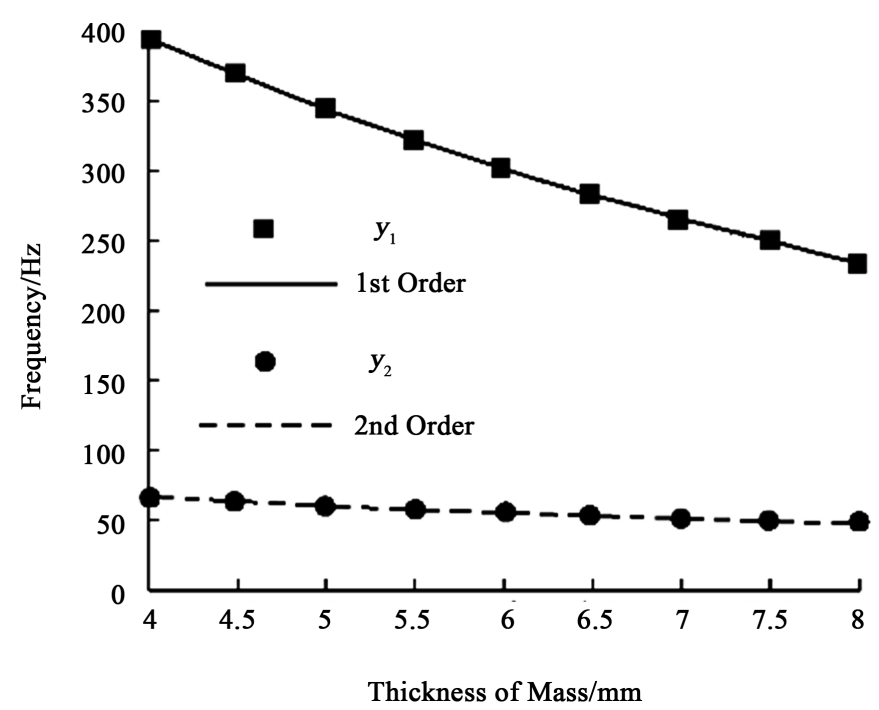

Figure 5. Relationship between mass thickness and natural frequency.

the one or two order natural frequency of the piezoelectric cantilever decreases with the increase of the thickness of the mass block, and the second order natural frequencies are below $100 \mathrm{~Hz}$. Thus, the natural frequency of the piezoelectric cantilever can be easily adjusted by adjusting the size of the mass block.

\subsection{Effect of Thickness of Piezoelectric Plates on Frequency and Output Voltage}

In order to analyze the influence of the thickness of piezoelectric ceramic plates on the natural frequency and output voltage of piezoelectric cantilever, the thickness of piezoelectric ceramic is set to $0.1-0.8 \mathrm{~mm}$, and the other parameters remain unchanged. The static force analysis and modal analysis of the piezoelectric cantilever beam are carried out at the free end (without mass) by applying the force of $0.3 \mathrm{~N}$. The analysis results are shown in Figure 6 and Figure 7 respectively.

The relation curves of synthesis Figure 6 and Figure 7 can be obtained on the basis of the low natural frequency of the piezoelectric structure, the piezoelectric plates' thickness corresponding to the maximum output voltage is selected.

\subsection{Effect of Substrate Length on Frequency and Output Voltage}

In order to analyze the effect of the metal elastic length on the natural frequency and output voltage of the piezoelectric cantilever, the length of the elastic substrate is set to $35-65 \mathrm{~mm}$, and the other parameters remain unchanged. The static force analysis and modal analysis of the piezoelectric cantilever beam are carried out at the free end (without mass) by applying the force of $0.3 \mathrm{~N}$. The analysis results are shown in Figure 8 and Figure 9 respectively.

The analysis of the relation curve between synthesis Figure 8 and Figure 9 shows that the increase of the length of the metal elastic substrate can not only reduce the natural frequency of the low-voltage structure, but also increase the 


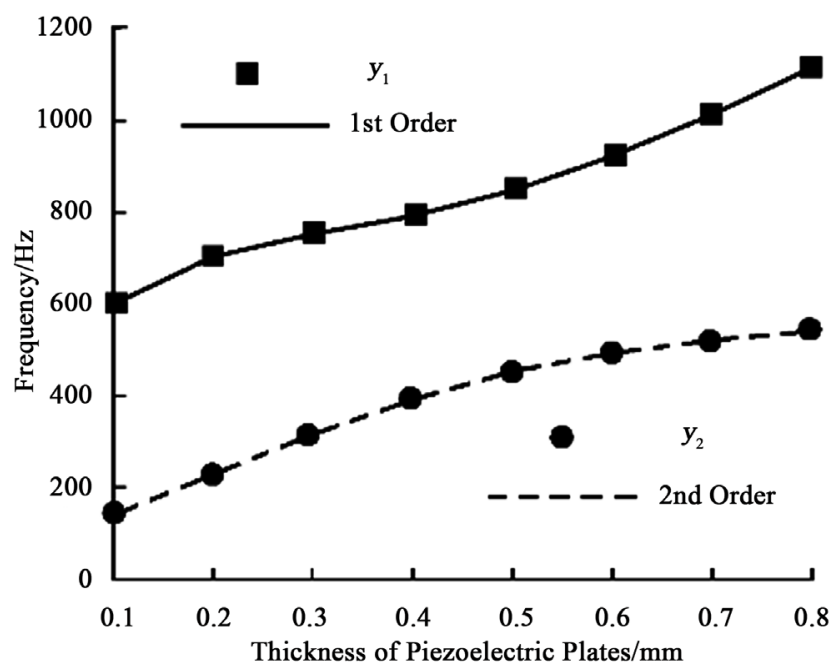

Figure 6. Relationship between thickness and frequency of piezoelectric plates.

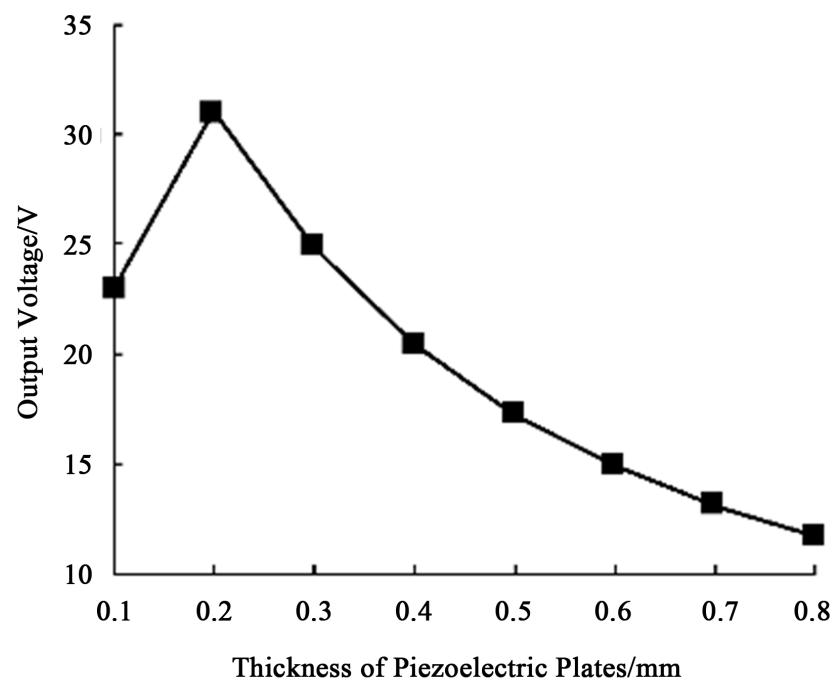

Figure 7. The relationship between piezoelectric plate thickness and output voltage.

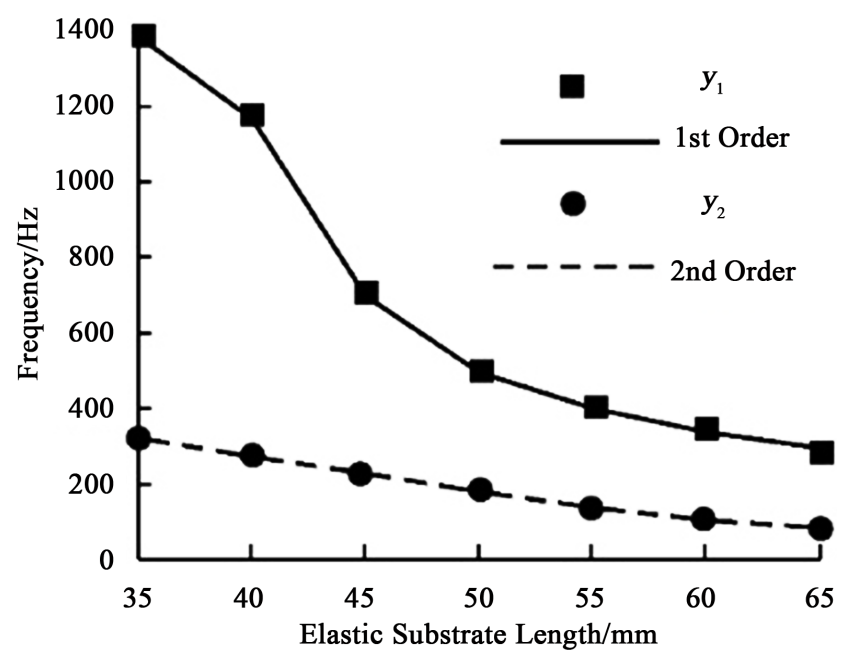

Figure 8. The relationship of frequency of substrate length. 


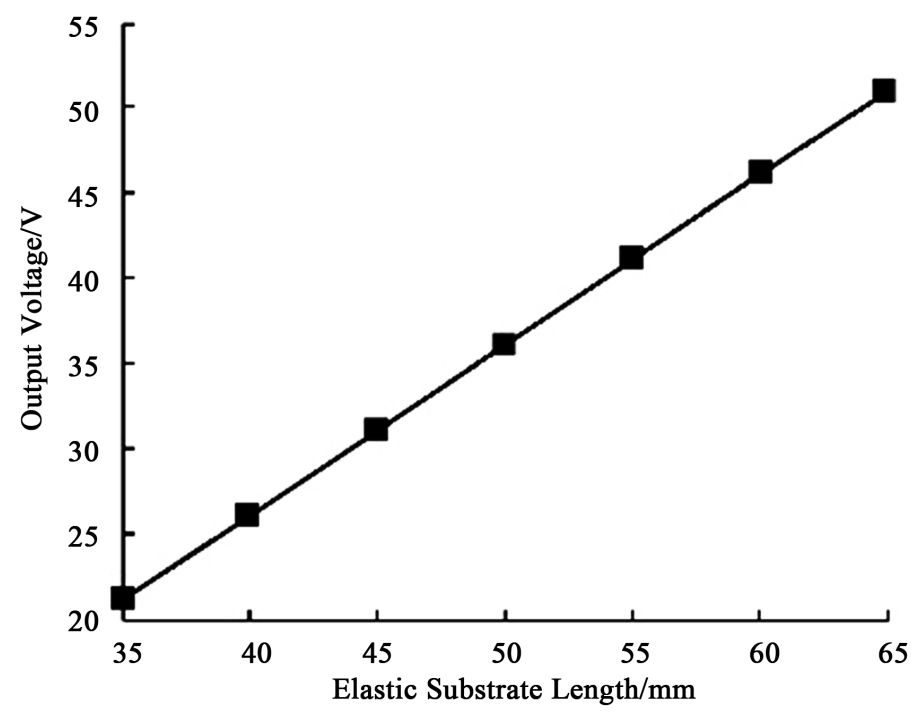

Figure 9. The Relationship between Substrate Length and output voltage.

output voltage. Therefore, in the actual design, long elastic substrate should be chosen as far as possible but within the scope of the stress permit.

\subsection{Effect of Substrate Thickness on Frequency and Output Voltage}

In order to analyze the influence of the thickness of the metal elastic substrate on the natural frequency and the output voltage of the piezoelectric cantilever, the thickness of the elastic substrate is set to $0.2-1 \mathrm{~mm}$, and the other parameters remain unchanged. The static force analysis and modal analysis of the piezoelectric structure are carried out at the free end (without mass) by applying $0.3 \mathrm{~N}$, and the analysis results are shown in Figure 10 and Figure 11 respectively.

The relation curve between synthesis Figure 10 and Figure 11 shows that the increase of the thickness of the elastic substrate not only increases the natural frequency of the piezoelectric structure, but also decreases the output voltage of the piezoelectric structure. Therefore, the thin elastic substrate should be chosen when the strength of the metal substrate is allowed.

\section{Experimental Test of Multidirectional Vibration Piezoelectric Generator}

\subsection{Experimental Test System}

Figure 12 is an experimental system for multidirectional piezoelectric generator, which consists of an 1) oscilloscope (DS5062C), 2) Sweep frequency signal generator (YE1311E), 3) multidirectional vibration piezoelectric generator, 4) The vibration exciter (JZK-2) and 5) the energy collection circuit. The sweep frequency signal generator can generate a sine wave signal with adjustable frequency and amplitude to drive the exciter to generate vibration. The multi-directional vibration piezoelectric power generation device outputs the voltage under the vibration of the exciter, and the output voltage is collected through the 


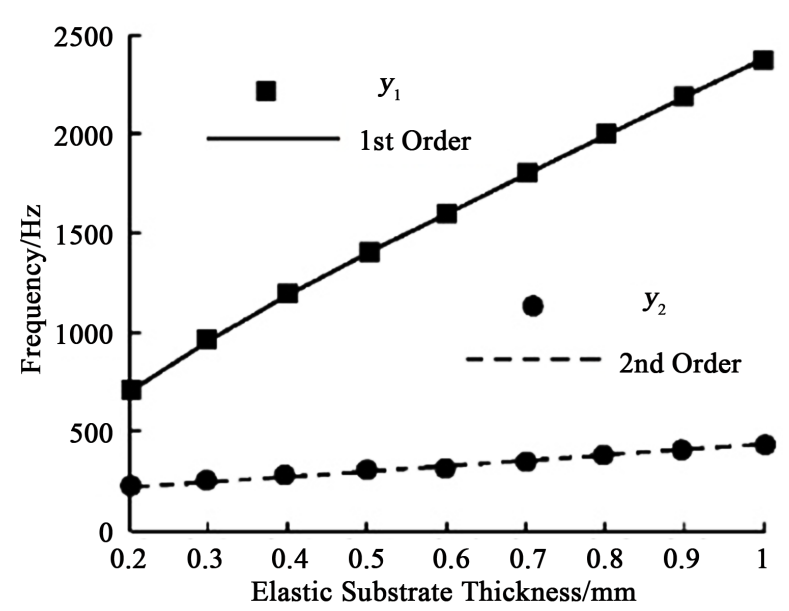

Figure 10. The relationship of frequency of substrate thickness.

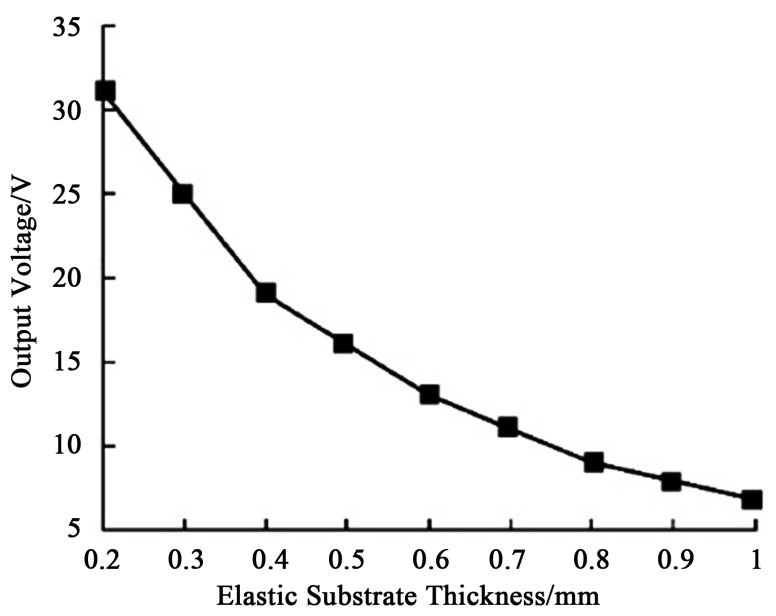

Figure 11. The relationship between substrate thickness and output voltage.

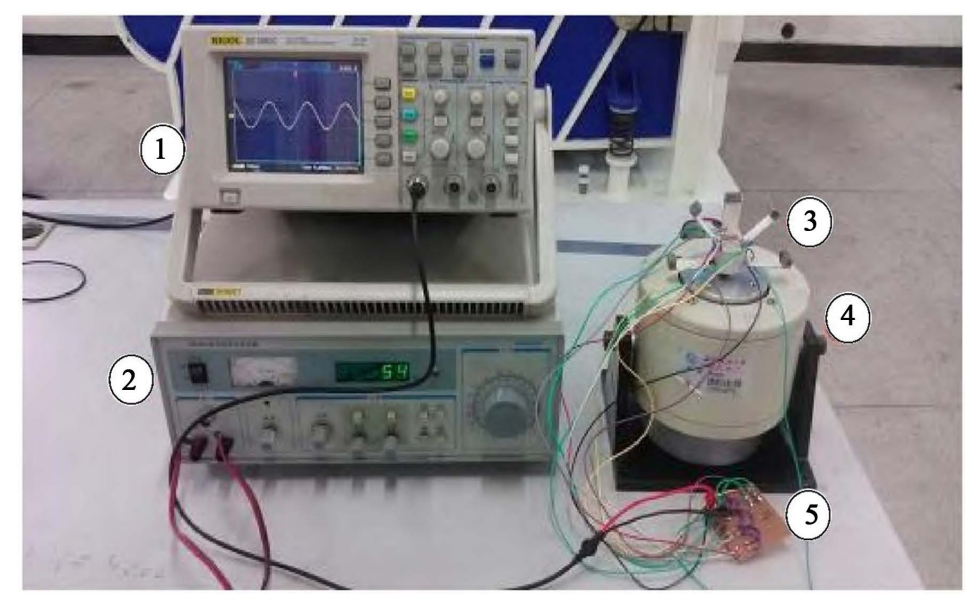

Figure 12. Experimental test system.

energy collection circuit. The oscilloscope is mainly used to observe the voltage signal generated by the multi-directional vibration piezoelectric power generation device. 


\subsection{Theoretical Load Output Analysis of Multidirectional Vibration Piezoelectric Generator}

The energy collection circuit of a cantilever beam in a single direction is a standard energy collection circuit, the theoretical load voltage and load power are respectively

$$
\begin{gathered}
V_{1, c u}=\frac{\omega R V_{1, n} C_{1}}{\omega R C_{1}+\frac{\pi}{2}} \\
P_{1, \text { cu }}=\frac{\omega^{2} R\left(V_{1, n} C_{1}\right)^{2}}{\left(\omega R C_{1}+\frac{\pi}{2}\right)^{2}}
\end{gathered}
$$

The energy collecting circuit of the multidirectional vibration device is connected in parallel by five standard energy collecting circuits. The theoretical load voltage and the load power are respectively

$$
\begin{gathered}
V_{r, c u}=\frac{\omega R\left(V_{1, n} C_{1}+V_{2, n} C_{2}+V_{3, n} C_{3}+V_{4, n} C_{4}+V_{5, n} C_{5}\right)}{\omega R\left(C_{1}+C_{2}+C_{3}+C_{4}+C_{5}\right)+\frac{\pi}{2}} \\
P_{r, \text { cu }}=\frac{\omega^{2} R\left(V_{1, n} C_{1}+V_{2, n} C_{2}+V_{3, n} C_{3}+V_{4, n} C_{4}+V_{5, n} C_{5}\right)^{2}}{\left[\omega R\left(C_{1}+C_{2}+C_{3}+C_{4}+C_{5}\right)+\frac{\pi}{2}\right]^{2}}
\end{gathered}
$$

where: $C_{1} \sim C_{5}$ and $V_{1, n} \sim V_{5, n}$, respectively, the equivalent capacitance and voltage of piezoelectric plates, in different directions. Through comparison and analysis, we can get:

$$
\begin{aligned}
& V_{r, \text { cu }}>V_{1, \text { cu }} \\
& P_{r, \text { cu }}>P_{1, \text { cu }}
\end{aligned}
$$

\subsection{Measurement of Natural Frequency and Open Circuit Voltage}

Figure 13 shows the relationship between the output voltage and the vibration frequency when a voltage amplitude of $2 \mathrm{~V}$ and $4 \mathrm{~V}$ is applied to a single piezoelectric cantilever beam. It is not difficult to see that when the frequency is $50 \mathrm{~Hz}$, the output voltage is the highest, which proves that the natural frequency of the piezoelectric cantilever beam is $50 \mathrm{~Hz}$. The simulation results show that the energy harvesting efficiency of the piezoelectric cantilever reaches the maximum value in all directions by finite element simulation analysis and optimization.

\subsection{Test Result Analysis of Multidirectional Vibration Piezoelectric Generator}

The theoretical load output is verified by applying different directions of excitation to the multidirectional piezoelectric device. When the $50 \mathrm{~Hz}$ vibration frequency is applied in the $\mathrm{Z}$ axis direction, Figure 14 and Figure 15 are the theoretical curves and experimental curves of the output voltage, output power and 


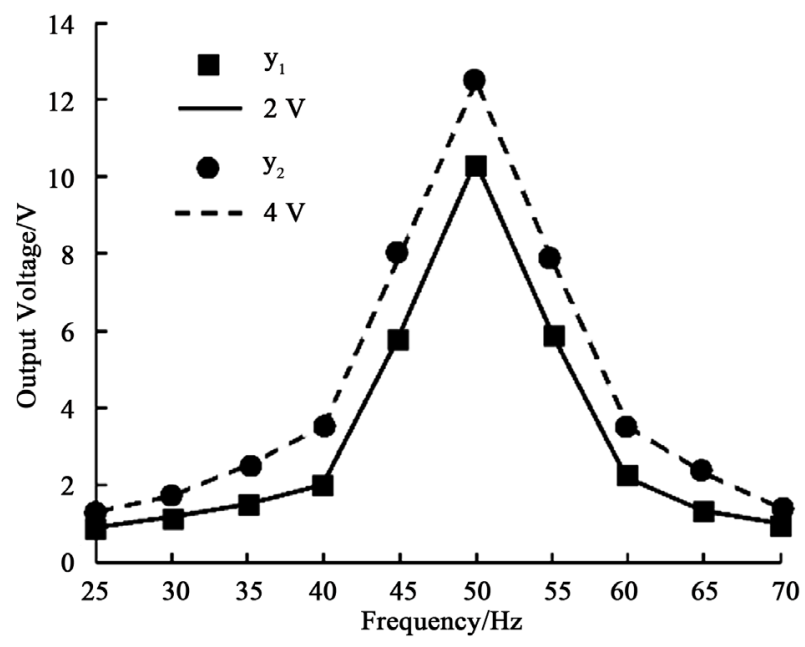

Figure 13. Relationship between output voltage and frequency of piezoelectric cantilever beam.

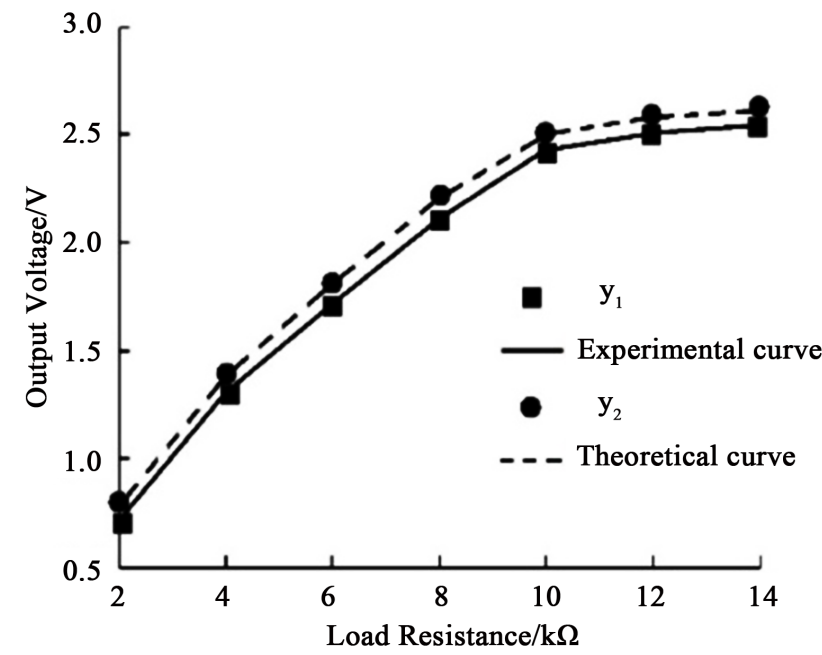

Figure 14. Relationship between output voltage and resistance of vibration device.

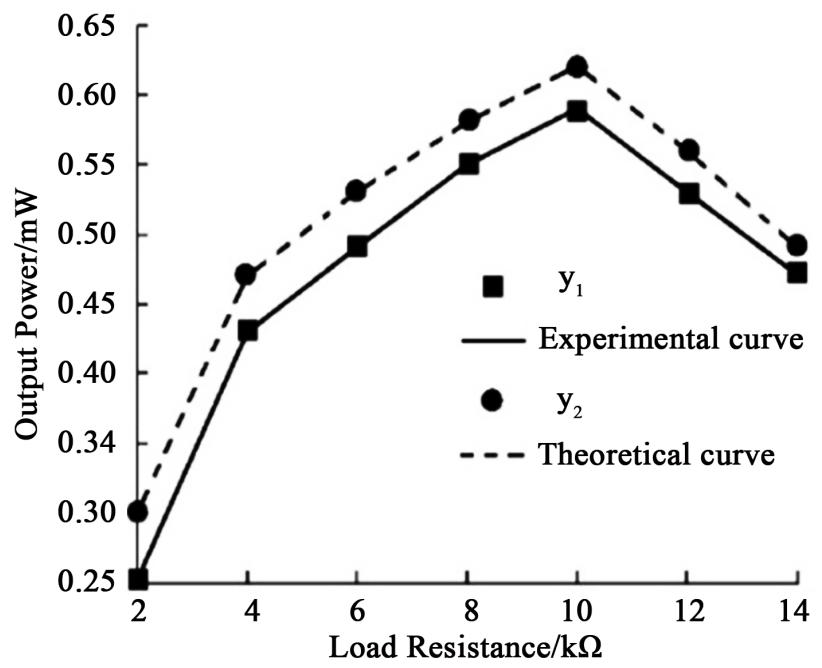

Figure 15. Relationship between output power and resistance of vibration device. 
load resistance of the device, respectively. It can be seen from the diagram that the variation trend of theoretical curve and experimental curve is basically the same, it is proved that the output voltage and the load power of the multidirectional vibration piezoelectric generator are larger than that of the unidirectional piezoelectric generator.

Figure 16 and Figure 17 are the curves of the output power and the vibration direction of the device when the vibration direction changes in different planes. From Figure 16 and Figure 17, the energy collection efficiency of the piezoelectric cantilever in this direction can reach the maximum when the vibration direction of the environment coincides with the piezoelectric cantilever beam; The

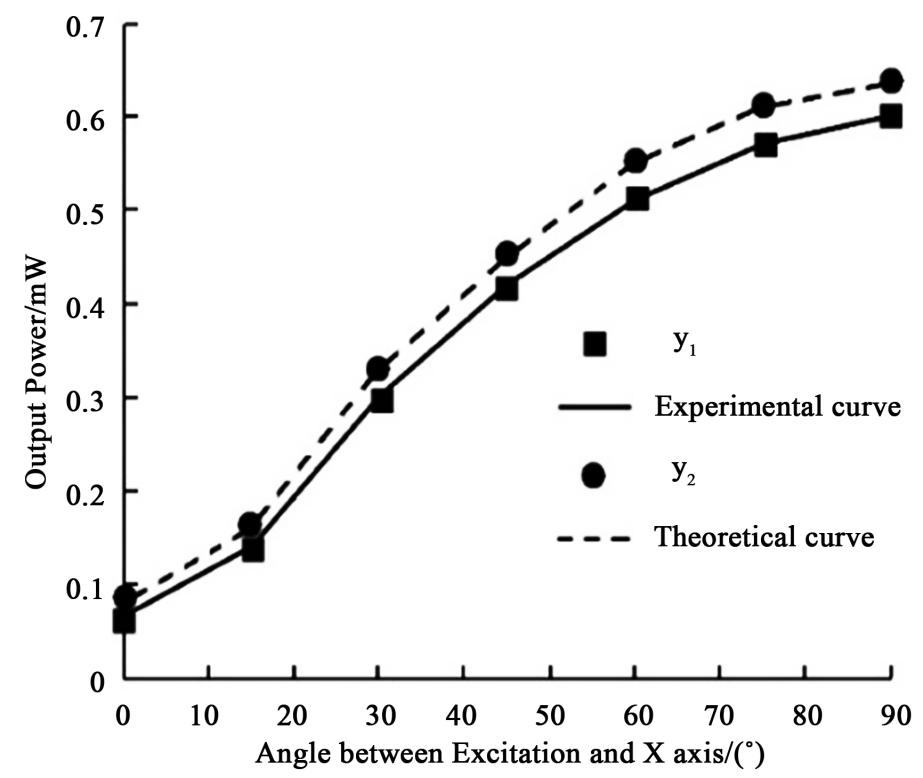

Figure 16. Output power of vibration direction varying in XZ plane.

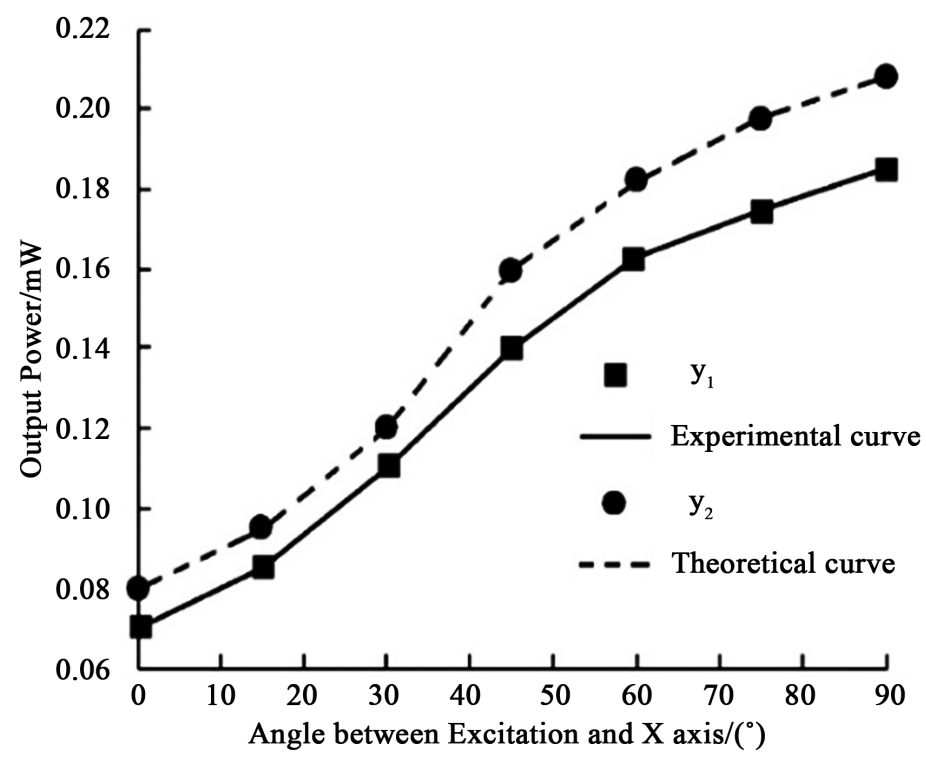

Figure 17. Output power of vibration direction varying in $\mathrm{XY}$ plane. 
cantilever beam with multiple directions in the piezoelectric device can largely eliminate the strong directional selectivity of the unidirectional piezoelectric generator, The vibration energy collection efficiency of the device reaches the highest level to adapt to the changeable vibration direction in the environment. Therefore, the multi directional vibration piezoelectric power generation device designed in this paper has higher energy collection efficiency than the traditional unidirectional piezoelectric generator.

\section{Conclusion}

The key technology of multidirectional vibration piezoelectric power generation is studied. The optimal model of piezoelectric power generation device is established by finite element simulation and analysis, the natural frequency of the device is consistent with the environmental frequency, which greatly improves the vibration energy collection efficiency of the piezoelectric device, and the energy collection in a plurality of directions breaks the limitation of the directional selectivity to the piezoelectric device. The problem of reducing the energy collection efficiency of unidirectional piezoelectric power generation devices due to the change of the vibration direction in the environment is eliminated. Finally, the feasibility and correctness of the design concept are proved through theoretical analysis and experimental comparison. The key technology of multidirectional vibration piezoelectric power generation can improve the energy collection efficiency of piezoelectric power generation device, and the technology is a green pollution-free technology, and has broad prospects for development.

\section{References}

[1] Gilbert, J.M. and Balouchi, F. (2008) Comparison of Energy Harvesting Systems for Wireless Sensor Networks. International Journal of Automation and Computing, $\mathbf{5}$, 334-347.

[2] Chalasani, S. and Conrad, J.M. (2008). A Survey of Energy Harvesting Sources for Embedded Systems. In Proceedings of IEEE Southeastcon 2008, 442-447. https://doi.org/10.1109/SECON.2008.4494336

[3] Saadon, S. and Side, O.F. (2011) A Review of Vibration Based MEMS Piezoelectric Energy Harvesters. Energy Conversion and Management, 52, 500-504.

[4] Habib, A., Arshad, A. and Khan, R. (2017) Distributed Renewable Energy under the Guidance of Price Autonomous Operation Technology. Smart Grid and Renewable Energy, 8, 305-324. https://doi.org/10.4236/sgre.2017.810020

[5] Kim, H.S., Kim, J.H. and Kim, J.A. (2011) Review of Piezoelectric Energy Harvesting Based on Vibration. International Journal of Precision Engineering and Manufacturing, 12, 1129-1141.

[6] Yan, Z. and He, Q. (2011) The Electrical Properties of Vibration Generator of J Piezoelectric Cantilever Beam Excitation Environment. Proceedings of CSEE, 31, 140-145. (in Chinese)

[7] Chen, R.W., Liu, X.J., et al. (2012) Self-Tuning Band Improved J Piezoelectric Vibration Generator. Journal of Nanjing University of Aeronautics \& Astronautics, 44, 327-332. 
[8] He, X.F., Wen, Z.Y. and Wen, Z. (2009) Modeling and Application of Piezoelectric Vibration Generator (English) J. Optics and Precision Engineering, 17, 1436-1441. (in Chinese)

[9] Kan, J.W., Yu, L., Wang, S.Y., et al. (2014) Rotating Magnetic Excitation Piezoelectric Cantilever Liang Fa Motor Performance Analysis and Test of J. Chinese Journal of Mechanical Engineering, 50, 144-149. (in Chinese)

[10] Qiu, Q.Q., Xiao, L.Y., Xin, S.Q., et al. (2011) A Miniature Piezoelectric Vibration Generator Design and Characteristic Analysis of J. Journal of Motor and Control, 15, 1-7. (in Chinese)

[11] Yan, Z., Wang, D.P., He, Q., et al. (2015) Piezoelectric Cantilever Beam of J Performance Optimization Type Vibration Generator. Journal of Materials, Sensing Technology, 28, 352-356. (in Chinese)

[12] Karami, M.A., Bilgen, O. and Inman, D.J. (2011) Experimental and Analytical Parametric Study of Single-Crystal Unimorph Beams for Vibration Energy Harvesting. IEEE Transactions on Ultrasonic, Ferroelectrics and Frequency Control, 58, 1508-1520.

[13] Ruan, Z.L., Gong, J.J., Cai, M.C., et al. (2013) Structural Design and Testing of a Butterfly Piezoelectric Generator with Multilayer Cantilever Beams. Advanced Materials Research, 655-657, 823-829.

https://doi.org/10.4028/www.scientific.net/AMR.655-657.823

[14] Xu, R., Lei, A. and Dahl-Petersen, C. (2012) Screen Printed PZT/PZT Thick Film Bimorph MEMS Cantilever Device for Vibration Energy Harvesting ... In: Sensors and Actuators A: Physical, 188, 383-388. https://doi.org/10.1016/j.sna.2011.12.035

[15] Yuan, J.B., Xie, T., et al. (2010) Xiao Biao Single, Composite Piezoelectric Cantilever Vibration Test Study Model and Generating J. Chinese Journal of Mechanical Engineering, 46, 87-92.

[16] Habib, A., Sou, C. and Ananta, A. (2017) Control Strategy of DC Link Voltage Flywheel Energy Storage for Non Grid Connected Wind Turbines Based on Fuzzy Control. Journal of Power and Energy Engineering, 5, 72-79. https://doi.org/10.4236/jpee.2017.511006

[17] Wang, J., Guo, J. and Guo, S. (2011) Piezoelectric Power Generation Technology Research. Piezoelectric and Acousto-Optic, 33, 394-398. (In Chinese)

[18] Deng, G., Tao, L., Chen, Z., et al. (2008) Since the Key Technology of Power Supply Analysis. Piezoelectric Ceramics Based on Automation, 27, 67-69. (In Chinese)

[19] Zhou, L. (1997) Mechanical Vibration Theory and Technology. Henan Science and Technology Press, Zhengzhou, 46-58. (In Chinese)

[20] Liu, H.C., Tay, C.J. and Quan, C.G. (2011) Piezoelectric MEMS Energy Harvester for Low Frequency Vibration with Wideband Operation Range and Steadily Increased Output Power. Journal of Microelectromechanical Systems, 20, 1131-1142. https://doi.org/10.1109/JMEMS.2011.2162488

[21] Yu, L. (2010) Study on Energy Transfer Structure of Multidirectional Vibration Energy Harvesting Based on Piezoelectric Materials. Nanjing University of Aeronautics \& Astronautics, Nanjing. (In Chinese)

[22] Wang, Q. (2010) Transducer Technology Based on Piezoelectric Effect. North Central University, Taiyuan.

[23] Liu, X.J. (2012) Multi Directional Piezoelectric Vibration Energy Harvesting Method and Key Techniques for Performance Optimization. Nanjing University of Aeronautics \& Astronautics, Nanjing. (in Chinese) 
[24] Tang, G., Liu, J., Yang, B. and Luo, J. (2012) Fabrication and Analysis of High-Performance Piezoelectric MEMS Generators. Journal of Micromechanics and Micro Engineering, 22, Article ID: 065017.

https://doi.org/10.1088/0960-1317/22/6/065017 